Conclusions: ABA appears to be effective in ILD associated-RA, including the pattern of poor prognosis (UIP).

References:

[1] Travis WD et al. J Respir Crit Care Med 2013 188:733-748.

Disclosure of Interest: None declared

DOI: 10.1136/annrheumdis-2017-eular.3492

\section{SAT0192 SARILUMAB SUPPRESSES THROMBOSIS-RELATED GENE EXPRESSION IN CIRCULATING BLOOD CELLS IN MTX-IR AND TNF-IR PATIENTS WITH ACTIVE RHEUMATOID ARTHRITIS}

C. Paccard $^{1}$, J. Msihid ${ }^{1}$, A. Brisacier ${ }^{1}$, A. Damask ${ }^{2}$, C. Paulding ${ }^{2}$,

M. Zilberstein ${ }^{3}$, A. Boyapati ${ }^{2} .{ }^{1}$ Sanofi R\&D, Chilly-Mazarin, France; ${ }^{2}$ Regeneron Pharmaceuticals, Inc, Tarrytown; ${ }^{3}$ Sanofi R\&D, Bridgewater, United States

Background: Bone and joint damage due to chronic inflammation in the synovium of patients with RA is mediated by IL-6 and other cytokines. IL-6 mediates effects inside the joint and systemically and is blocked by sarilumab, a human mAb blocking the IL-6R?. Safety and efficacy of SC sarilumab (150 or $200 \mathrm{mg} \mathrm{q2w}$ ) was evaluated in combination with MTX in patients with RA and inadequate response (IR) to MTX (MOBILITY; NCT01061736) or csDMARDs in patients with RA and IR or intolerance to $\geq 1$ TNFi (TARGET; NCT01709578).

Objectives: To compare gene expression patterns in circulating blood cells after treatment with sarilumab vs placebo in patients from MOBILITY and TARGET.

Methods: Total RNA was isolated from whole blood collected at baseline (predose) and wk 2 posttreatment in patients from MOBILITY (placebo, $n=58$; sarilumab $150 \mathrm{mg}$ q2w, $\mathrm{n}=60$; sarilumab $200 \mathrm{mg}$ q2w, $\mathrm{n}=46$ ) and TARGET (placebo, $n=19$; sarilumab $150 \mathrm{mg}$ q2w, $n=27$; sarilumab $200 \mathrm{mg}$ q2w, $n=16$ ). Gene expression data were generated using microarray analyses (Agilent $8 \times$ $60 \mathrm{k}$ platform). Data were processed using the limma package ( $\mathrm{R}$ Bioconductor). Background correction and quantile normalization were performed and probes with low expression were filtered; 48,109 probes were analyzed. Effect of sarilumab vs placebo on gene expression was assessed in each study using linear mixed models. $P$ values were adjusted using the Benjamini-Hochberg procedure to control false discovery rate (FDR; $5 \%$ threshold).

Results: After $P$ value adjustment to control FDR at $5 \%, 2$ genes implicated in thrombosis and atherosclerosis (thrombomodulin [THBD] and platelet endothelial cell adhesion molecule 1 [PECAM-1]) were downregulated after treatment with sarilumab $200 \mathrm{mg}$ q2 $\mathrm{w}$ vs placebo in both studies (Table). These genes decreased with a fold-change $(\mathrm{FC}) \leq 0.8$ in both studies $(P<0.001)$. An additional gene associated with coagulation, von Willebrand factor (vWF), was significantly decreased in MTX-IR but not TNF-IR patients. Numerical decreases in gene expression between sarilumab $150 \mathrm{mg}$ q2w and placebo did not reach significance.

Table 1. Select Genes Regulated by Sarilumab $200 \mathrm{mg}$ q2w at Week 2

\begin{tabular}{|c|c|c|c|c|}
\hline \multirow[b]{2}{*}{ Gene } & \multicolumn{2}{|c|}{$\begin{array}{c}\text { MOBILITY } \\
\text { Sarilumab } 200 \text { mg q2w + MTX }\end{array}$} & \multicolumn{2}{|c|}{$\begin{array}{c}\text { TARGET } \\
\text { Sarilumab } 200 \mathrm{mg} \text { q2 } w+\text { csDMARDs }\end{array}$} \\
\hline & $P$ value & Fold reduction $^{a}$ & $P$ value & Fold reduction ${ }^{a}$ \\
\hline THBD & $6.3 \times 10^{-7}$ & 0.67 & $3.9 \times 10^{-6}$ & 0.58 \\
\hline PECAM-1 & $1.6 \times 10^{-9}$ & 0.78 & $2.1 \times 10^{-4}$ & 0.78 \\
\hline vWF & $1.3 \times 10^{-10}$ & 0.58 & 0.55 & 0.91 \\
\hline
\end{tabular}

a Fold reduction vs placebo.

Conclusions: In patients with active RA, sarilumab may decrease thrombosisrelated gene expression in circulating immune cells. Additional analysis of the serum levels of thrombosis risk proteins is needed to test the hypothesis that sarilumab treatment decreases levels of thrombosis risk factors.

Acknowledgements: This study was sponsored by Sanofi Genzyme and Regeneron Pharmaceuticals, Inc. Editorial support was provided by MedThink SciCom and funded by Sanofi Genzyme and Regeneron Pharmaceuticals, Inc. Disclosure of Interest: C. Paccard Shareholder of: Sanofi R\&D, Employee of: Sanofi R\&D, J. Msihid Shareholder of: Sanofi R\&D, Employee of: Sanofi R\&D, A. Brisacier Shareholder of: Sanofi R\&D, Employee of: Sanofi R\&D, A. Damask Shareholder of: Regeneron Pharmaceuticals, Inc, Employee of: Regeneron Pharmaceuticals, Inc, C. Paulding Shareholder of: Regeneron Pharmaceuticals, Inc, Employee of: Regeneron Pharmaceuticals, Inc, M. Zilberstein Shareholder of: Sanofi R\&D, Employee of: Sanofi R\&D, A. Boyapati Shareholder of: Regeneron Pharmaceuticals, Inc, Employee of: Regeneron Pharmaceuticals, Inc DOI: 10.1136/annrheumdis-2017-eular.5183

\section{SAT0193 RA PATIENTS WITH INFLAMMATORY ANEMIA BENEFIT FROM INCREASED HEMOGLOBIN AND DECREASED FATIGUE UNDER TOCILIZUMAB THERAPY}

C. Specker $^{1}$, H. Kellner ${ }^{2}$, P. Kästner ${ }^{3}$, C. Volberg ${ }^{4}$, V. Braunewell ${ }^{5}$

I. Schwarze ${ }^{6}$, M. Aringer ${ }^{7}$, M. Sieburg ${ }^{8}$, M.W. Hofmann ${ }^{9}$, J.P. Flacke ${ }^{10}$,

H.-P. Tony ${ }^{11}$, G. Fliedner ${ }^{12} .{ }^{1}$ Kliniken Essen Süd, Essen; ${ }^{2}$ Schwerpunktpraxis für Rheumatologie und Gastroenterologie, München; ${ }^{3}$ MVZ Ambulantes Rheumazentrum Erfurt, Erfurt; ${ }^{4}$ Rheumazentrum Neuss, Neuss;

${ }^{5}$ Schwerpunktpraxis Rheumatologie, Mönchengladbach; ${ }^{6}$ Praxis für Internistische Rheumatologie, Leipzig; ${ }^{7}$ Medizinische Klinik III, Rheumatologie,

Technischen Universität Dresden, Dresden, ${ }^{8}$ Rheumatologische
Gemeinschaftspraxis, Magdeburg: ${ }^{9}$ Rheumatologie, Chugai Pharma Europe Ltd., Frankfurt; ${ }^{10}$ Rheumatologie, Roche Pharma AG, Grenzach-Wyhlen

${ }^{11}$ Rheumatologie/Immunologie der Medizinischen Klinik und Poliklinik II, Universitaetsklinikum Wuerzburg, Wuerzburg; ${ }^{12}$ Rheumapraxis, Osnabrueck, Germany

Background: According to WHO definition approximately $15 \%$ of all patients with rheumatoid arthritis (RA) suffer from anemia (hemoglobin $<13 \mathrm{~g} / \mathrm{dl}$ for men and $<12 \mathrm{~g} / \mathrm{dl}$ for women). Interleukin 6 (IL-6) takes an active part in the pathogenesis of this inflammatory anemia.

Objectives: The 6th interim analysis of the non-interventional ICHIBAN study (NCT01194401) evaluated the occurrence of inflammatory anemia, characterized the patient population with anemia, and observed the response during intravenous Tocilizumab therapy (TCZ i.v.). Patients were subgrouped according to their anemic/non-anemic status at baseline.

Methods: Since 2010 the ICHIBAN study collects clinical data of the routine use of TCZ i.v. in RA patients. The observation period for each patient is up to two years. At the due date of the current interim analysis (Dec 10, 2015) 2999 patients were enrolled. 902 patients have completed the maximal 104 weeks observation period (Group W104).

Results: At baseline, the proportion of patients with anemia (acc. to WHO definition) was $21.4 \%$ (men) and $22.0 \%$ (women) in the group W104.

On comparison, RA patients with anemia showed, amongst others, increased inflammation parameters, a higher disease activity and higher rates of comorbidities. Already after 4 weeks with TCZ i.v. the proportion of patients with anemia improved to $12.1 \%$ (men) and $12.7 \%$ (women). After 104 weeks therapy the proportion of patients with anemia reduced further to $7.4 \%$ (men) and $8.4 \%$ (women). The relevant response parameters and laboratory values are shown in Table 1

Despite the higher disease activity at baseline for anemic patients, the benefit was comparable for patients with and without anemia. DAS28-ESR values decreased on average by 2.9 (women) and 3.1 (men) in RA patients with anemia and by 2.7 (women) and 2.8 (men) in RA patients without, resulting in similar disease scores at the end of the observational period.

The effectiveness of TCZ i.v. was also confirmed by patient reported outcomes (PROs) via visual analogue scales (VAS). In particular, a reduction of the intensity of pain $(>50 \%)$ and a reduction of fatigue (>38\%) was observed (Table 1$)$

Table 1 Treatment response to $\mathrm{TCZ}$ in anemic patients (at baseline)

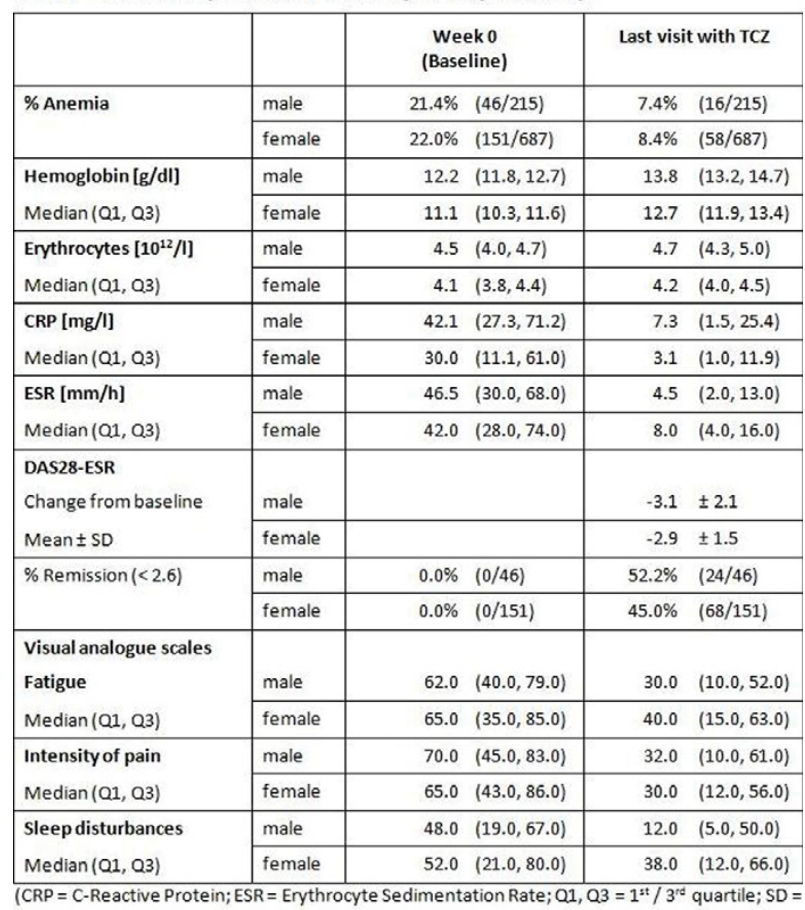

standard deviation

Conclusions: At start of therapy, approximately one out of five patients documented in ICHIBAN showed anemia according to the WHO definition. During TCZ i.v. therapy a noticeable decrease in the rate of anemia and improved hemoglobin values were observed. These effects can already be seen after four weeks of treatment and continue up to the end of this study (i.e. 2 years). Despite the higher burden of disease at baseline in RA patients with anemia, TCZ i.v. therapy resulted in good clinical response rates and PROs.

Acknowledgements: We would like to thank all patients, their families, the investigators and the nurses who participated in this trial. This research was funded by Roche Pharma AG, Germany, and Chugai Pharma Europe Ltd., Germany. Support for third-party writing assistance for this abstract presentation 